\title{
Molecular diversity of rumen bacterial communities from tannin-rich and fiber-rich forage fed domestic Sika deer (Cervus nippon) in China
}

Zhi Peng Li ${ }^{1}$, Han Lu Liu', Guang Yu Li ${ }^{1 *}$, Kun Bao', Kai Ying Wang ${ }^{1}$, Chao Xu' ${ }^{1}$ Yi Feng Yang ${ }^{1}$, Fu He Yang ${ }^{1}$ and André-Denis G Wright ${ }^{2}$

\begin{abstract}
Background: Sika deer (Cervus nippon) have different dietary preferences to other ruminants and are tolerant to tannin-rich plants. Because the rumen bacteria in domestic Sika deer have not been comprehensively studied, it is important to investigate its rumen bacterial population in order to understand its gut health and to improve the productivity of domestic Sika deer.

Results: The rumen bacterial diversity in domestic Sika deer (Cervus nippon) fed oak leaves- (OL group) and corn stalks-based diets (CS group) were elucidated using 16S rRNA gene libraries and denaturing gradient gel electrophoresis (DGGE). Overall, 239 sequences were examined from the two groups, 139 clones from the OL group were assigned to 57 operational taxonomic units (OTUs) and 100 sequences from the CS group were divided into 50 OTUs. Prevotella-like sequences belonging to the phylum Bacteroidetes were the dominant bacteria in both groups (97.2\% OL and 77\% CS), and sequences related to Prevotella brevis were present in both groups. However, Prevotella shahii-like, Prevotella veroralis-like, Prevotella albensis-like, and Prevotella salivae-like sequences were abundant in the $\mathrm{OL}$ group compared to those in the CS group, while Succinivibrio dextrinosolvens-like and Prevotella ruminicola-like sequences were prevalent in the CS group. PCR-DGGE showed that bacterial communities clustered with respect to diets and the genus Prevotella was the dominant bacteria in the rumen of domestic Sika deer. However, the distribution of genus Prevotella from two groups was apparent. In addition, other fibrolytic bacteria, such as Clostridium populeti and Eubacterium cellulosolvens were found in the rumen of domestic Sika deer.

Conclusions: The rumen of domestic Sika deer harbored unique bacteria which may represent novel species. The bacterial composition appeared to be affected by diet, and sequences related to Prevotella spp. may represent new species that may be related to the degradation of fiber biomass or tannins. Moreover, the mechanism and biological functions of Prevotella spp. in the rumen ecosystem, and synergistic interactions with other microorganisms should be noticed.
\end{abstract}

Keywords: Ecology, Prevotella, Fiber, Tannin

\footnotetext{
* Correspondence: tcslgy@126.com

'Department of Economical Animal Nutrition and Feed Science, Institute of

Special Animal and Plant Sciences, Chinese Academy of Agricultural

Sciences, JiLin, China

Full list of author information is available at the end of the article
} 


\section{Background}

Sika deer (Cervus nippon) represent the most ancient and primitive members of the genus Cervus because of the simple structure of their antlers, which is very distinct from those of reindeer. Velvet antlers are one of the main products from Sika deer, and are used in traditional Chinese medicine. In addition, Sika deer yield high quality meat and skin. Domestication of Sika deer began much later than for other ruminants. At present, the number of domesticated Sika deer in China is approximately 550,000 head, most of which are distributed in northwestern China.

In nature, Sika deer graze a wide range of forage types, such as Amur grape, elm, maple, bamboo and some toxic species including Chinese Stellera roots and large flowered larkspurs. Moreover, grazing Sika deer have been observed to prefer tannin-rich plants, such as oak leaves. Similar behavior has also been observed in wild Sika deer (Cervus nippon yesoensis) inhabiting the Shiretoko Peninsula of Hokkaido Island in Japan, and in the roe deer (Capreolus capreolus) [1,2]. However, domesticated Sika deer held in captivity are commonly fed corn stalks containing a much higher fibrous content. Like other ruminants, Sika deer depend on the rumen for fermentation that involves the conversion of plant fiber to volatile fatty acids. This involves a diverse and dense array of microorganisms, including bacteria, fungi, archaea and protozoa [3]. Among these microorganisms bacterial populations have been extensively studied for many years since rumen bacteria have important roles in the efficient degradation of plant biomass and detoxification of secondary compounds in plants [1,4-7]. This has led to a variety of studies investigating rumen bacterial structure have been conducted on domestic cows, sheep, yak, Reindeer in Norway and wild Sika deer in Japan [4,5,8-10]. Moreover, rumen bacterial communities are affected by the host and diet $[11,12]$. To our knowledge, very little is known about the rumen bacterial community of domesticated Sika deer in China. A comprehensive understanding of bacterial ecology in the rumen of domesticated Sika deer is necessary to increase the efficiency of fiber digestion and to improve the productivity of velvet antlers.

Thus, we hypotheses the bacterial communities in the rumen of domesticated Sika deer may be unique. And the objectives of the present study were: (1) to describe the bacterial diversity in the rumen from domesticated Sika deer ingesting different diets based on 16S rRNA gene sequence libraries and PCR-DGGE; and (2) to compare the unique rumen bacterial populations of domesticated Sika deer ingesting tannin-rich and fiber-rich materials.

\section{Results}

Comparative analysis of 16S rRNA gene libraries from two groups

A total of 239 non-chimeric sequences were analyzed, 139 sequences from the OL 16S rRNA clone library and
100 sequences from the CS clone library. The two rumen bacterial populations were distinct according to the RDP classifier tool at a confidence threshold of $80 \%$ (Figure 1). Within the two groups, members of the phylum Bacteroidetes were the predominant bacteria (99.3\% and $85 \%$ of clones in the OL and CS groups, respectively). Domesticated Sika deer consuming corn stalks has Firmicutes present whereas they were not found in oak leaves fed domesticated Sika deer. Similarly, Proteobacteria were more expressed in corn stalks than oak leaves diets. The Chao1 (114.2 vs 143.5) and Shannon-Wiener (3.5 vs 3.7) indices of domesticated Sika deer consuming oak leaves were decreased compared to those feeding on corn stalks (Table 1). Moreover, the Libshuff analysis also showed that the bacterial communities between two diets were significantly differed $(P<0.0001)$. Rarefaction curves at $3 \%$ distance levels revealed $74 \%$ and $66 \%$ coverage for the OL and CS groups, respectively (Figure 2).

Using the software program MOTHUR and a sequence identity criterion cut off of $97 \%$, the $139 \mathrm{OL}$ clone sequences were assigned to 57 OTUs and the 100 CS clone sequences were assigned to 50 OTUs (Table 1). To determine the nearest valid related species, the $16 \mathrm{~S}$ rRNA gene sequences were compared using GenBank's Basic Local Alignment Search Tool (BLAST). Within the OL library, 53 of the 57 OTUs (i.e. $97.2 \%$ of clones) had $85 \%$ or greater sequence identities to genus Prevotella (Table 2). Within these OTUs, 23 OTUs (38.1\% of clones) showed $87-92 \%$ sequence identities to $P$. brevis, 11 OTUs (16.5\% of clones) had $86-90 \%$ sequence identities to $P$. shahii, 3 OTUs (23.8\% of clones) had $91-92 \%$ sequence identities to $P$. veroralis, 6 OTUs $(12.3 \%$ of clones) had distant sequence identities to $P$. salivae, and the remaining 9 OTUs (6.5\% of clones) showed sequence identities to several Prevotella species including $P$. albensis, $P$. dentalis, $P$. ruminicola, $P$. multiformis, P. stercorea, P. bryantii and P. copri (Table 2). Of the remaining 4 OTUs (of the 57 total OTUs), 2 OTUs (1.4\% of clones) were distantly related (85\%) to Alistipes shahii, 1 OTU ( $0.7 \%$ of clones) had $84 \%$ identity to

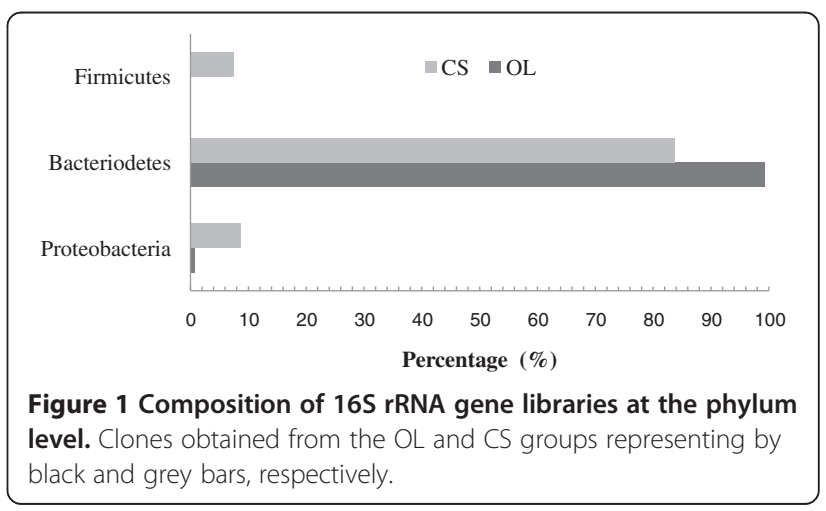


Table 1 Number of OTUs, diversity and coverage at 3\% distance level using the MOTHUR platform

\begin{tabular}{cccccc}
\hline Groups & Clones & OTUs & Chao $\mathbf{1}^{\mathbf{a}}$ & $\begin{array}{c}\text { Shannon- } \\
\text { Wiener }^{\mathbf{b}}\end{array}$ & Coverage \\
\hline OL & 139 & 57 & $\begin{array}{c}114.2 \\
(81.1,192.8)\end{array}$ & $\begin{array}{c}3.5 \\
(3.3,3.7)\end{array}$ & 0.74 \\
& & & 143.5 & $\begin{array}{c}3.7 \\
(85.8,294.1)\end{array}$ & 0.66 \\
CS & 100 & 50 & $(3.4,3.8)$ & \\
& & & &
\end{tabular}

a Chao1 is a nonparametric estimator of the richness in a sample. It is based on the number of rare ribotypes (singletons and doublets) and used to predict the species richness.

b The Shannon-Wiener index is a nonparametric diversity index that combines estimates of richness (total numbers of ribotypes) and evenness (relative abundance of each ribotype) suggesting diversity. It takes into account the abundance of individual taxa and can be used as an overall indicator of the level of diversity in a sample.

Barnesiella intestinihominis, and 1 OTU (0.7\% of clones) had $97 \%$ sequence identity to $S$. dextrinosolvens.

Within the CS clone library, 36 of the 50 OTUs were 85-98\% related to species belonging to genus Prevotella. Within these 36 OTUs, only one OTU (2\% of clones) had $>97 \%$ sequence identity to $P$. brevis, 14 OTUs $(36 \%$ of clones) had $90-93 \%$ identity to $P$. brevis and 11 OTUs (27\% of clones) had $91-95 \%$ identity to $P$. ruminicola making them the dominant bacterial species, whereas the remaining 10 OTUs (12\% of clones) exhibited distant sequence identity to $P$. shahii, $P$. veroralis, $P$. albensis, $P$. salivae and $P$. dentalis. Of the remaining 14 OTUs (of the 50 total), 3 OTUs ( $3 \%$ of clones) were distantly related (89\%) to Paraprevotella clara, 1 OTU (9\% of clones) showed $97 \%$ identity to $S$. dextrinosolvens, 3 OTUs (3\% of clones) had 90-95\% identity to Ruminococcus bromii, 2 OTUs (2\% of clones) had $84 \%$ identity to Parabacteroides merdae, 1 OTU ( $1 \%$ of clones) was $86 \%$ related to Clostridium aldrichii, and 1 OTU (1\% of clones) was $91 \%$ related to Clostridium bolteae, 4 other OTUs (4\% of clones) showed distant sequence identities to Roseburia hominis, Proteiniphilum acetatigenes, A. shahii and Sporanaerobacter acetigenes, respectively.

Overall, phylogenetic analysis revealed that the 107 OTUs were divided into six distinct phylogenetic groups

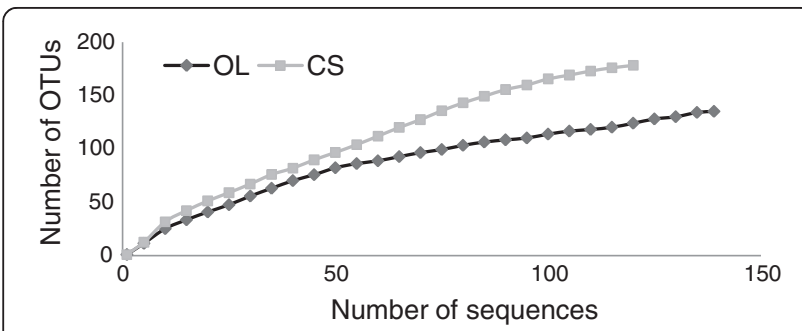

Figure 2 Rarefaction curves for bacterial 16S rRNA gene libraries. Dark and gray represent Sika deer feeding on oak leaves-based (OL group) and corn stalks-based (CS group) diets, respectively. Rarefaction curves were generated from the platform MOTHUR using the furthest neighbor method.
(Figure 3).In addition, the comparison between Norwegian reindeer, Svalbard reindeer and domesticated Sika deer at community level with Fast Unifrac [13], which analyze phylogenetic lineages, showed that the bacterial composition in the rumen of domesticated Sika deer fed oak leaves based diets was more similar to that of domesticated Sika deer fed corn stalks based diets, and differed from Svalbard reindeer and Norwegian reindeer (Figure 4). However, there were also shared bacterial communities between domestic Sika deer and Reindeer.

\section{Rumen bacterial diversity based on the PCR-DGGE profile}

PCR-DGGE banding profiles showed that the bacterial communities clustered with respect to diets (Figure 5). However, considerable animal-to-animal variation was also observed. A distinct difference in the bacterial structure was observed between two diets. By comparing the PCR-DGGE profiles between the two diets, the number of DGGE bands from CS group was considerably abundant compared to those from OL group (Figure 5). There were also several bands that were common for all domestic Sika deer.

In total, 47 dominant bands were excised from the PCR-DGGE profile and sequenced, of which 20 and 27 bands obtained from the OL and CS groups, respectively (see Additional file 1). Sequences from the excised bands from the OL group belonged to the phyla Firmicutes, Bacteroidetes and Proteobacteria, whereas DGGE sequences from the CS group belonged to the phyla Firmicutes, Bacteroidetes, Proteobacteria and Synergistetes. Among the 47 bands, 13 bands in two groups were identified as known species based on $\geq 97 \%$ sequence similarity (Table 3). Bands O-1, C-3 and C-5 showed $\geq 98 \%$ similarity with known species of C. populeti 743A. Bands O-3 and O-18 were identified as Streptococcus pasteurianus CIP 107122, while bands O-9 and C-14 showed 98\% similarity with of Eubacterium cellulosolvens 6. Band O-12 displayed 97\% similarity with known species of Moryella indoligenes AIP 220.04, and band O-13 showed specieslevel sequence similarity to Pseudobutyrivibrio ruminis DSM9787. Bands O-10 and C-10 displayed 98\% similarity to Succinivibrio dextrinosolvens 0554, while bands C-18 and $\mathrm{C}-1$ had $98 \%$ sequence similarity to Coprococcus eutactus ATCC 27759 and Prevotella ruminicola ATCC 19189, respectively. Moreover, band C-21 had the 93\% similarity with known species of Eubacterium ruminantium GA 195. Bands C-13 and C-22 were distantly related to Galbibacter mesophilus Mok-17 with $88 \%$ and 91\% similarity, respectively. Band C-24 displayed $88 \%$ similarity with Capnocytophaga cynodegmi CIP 103937, and band C-27 showed 94\% similarity with known species of Bacteroides uniformis JCM 5828. Bands C-19 and C-20 had $92 \%$ similarity with known species of Dethiosulfovibrio acidaminovorans sr15. The remaining 30 bands from two 
Table 2 Comparison of 16S rRNA gene libraries between the OL and CS groups

\begin{tabular}{|c|c|c|c|c|c|c|c|c|c|}
\hline \multicolumn{5}{|c|}{ OL group } & \multicolumn{5}{|c|}{ CS group } \\
\hline Phylotype & Clones $^{a}$ & OTU\# & Nearest Taxon & $\%^{b}$ & Phylotype & Clones $^{a}$ & OTU\# & Nearest Taxon & $\%^{b}$ \\
\hline SDMOL10 & 1 & 1 & P. brevis & 89 & SDCS52 & 1 & 1 & P. brevis & 90 \\
\hline SDMOL20 & 1 & 2 & P. brevis & 89 & SDCS61 & 1 & 2 & P. brevis & 90 \\
\hline SDMOL48 & 1 & 3 & P. brevis & 89 & SDCS69 & 6 & 3 & P. brevis & 90 \\
\hline SDMOL69 & 1 & 4 & P. brevis & 89 & SDCS71 & 2 & 4 & P. brevis & 90 \\
\hline SDMOL96 & 10 & 5 & P. brevis & 89 & SDCS1 & 3 & 5 & P. brevis & 91 \\
\hline SDMOL29 & 2 & 6 & P. brevis & 89 & SDCS74 & 1 & 6 & P. brevis & 91 \\
\hline SDMOL33 & 1 & 7 & P. brevis & 90 & SDCS80 & 1 & 7 & P. brevis & 91 \\
\hline SDMOL38 & 1 & 8 & P. brevis & 90 & SDCS14 & 1 & 8 & P. brevis & 92 \\
\hline SDMOL80 & 1 & 9 & P. brevis & 90 & SDCS40 & 4 & 9 & P. brevis & 92 \\
\hline SDMOL91 & 1 & 10 & P. brevis & 90 & SDCS49 & 3 & 10 & P. brevis & 92 \\
\hline SDMOL107 & 1 & 11 & P. brevis & 90 & SDCS41 & 5 & 11 & P. brevis & 92 \\
\hline SDMOL108 & 1 & 12 & P. brevis & 90 & SDCS5 & 1 & 12 & P. brevis & 93 \\
\hline SDMOL115 & 2 & 13 & P. brevis & 90 & SDCS8 & 1 & 13 & P. brevis & 93 \\
\hline SDMOL120 & 2 & 14 & P. brevis & 90 & SDCS93 & 6 & 14 & P. brevis & 93 \\
\hline SDMOL4 & 1 & 15 & P. brevis & 91 & SDCS16 & 2 & 15 & P. brevis & 98 \\
\hline SDMOL27 & 2 & 16 & P. brevis & 91 & SDCS85 & 1 & 16 & P. salivae & 90 \\
\hline SDMOL32 & 1 & 17 & P. brevis & 91 & SDCS48 & 2 & 17 & P. salivae & 91 \\
\hline SDMOL84 & 2 & 18 & P. brevis & 91 & SDCS2 & 1 & 18 & P. salivae & 92 \\
\hline SDMOL92 & 2 & 19 & P. brevis & 91 & SDCS90 & 1 & 19 & P. ruminicola & 91 \\
\hline SDMOL17 & 5 & 20 & P. brevis & 92 & SDCS98 & 5 & 20 & P. ruminicola & 92 \\
\hline SDMOL55 & 1 & 21 & P. brevis & 92 & SDCS53 & 1 & 21 & P. ruminicola & 93 \\
\hline SDMOL68 & 8 & 22 & P. brevis & 92 & SDCS54 & 3 & 22 & P. ruminicola & 93 \\
\hline SDMOL110 & 4 & 23 & P. brevis & 92 & SDCS78 & 1 & 23 & P. ruminicola & 93 \\
\hline SDMOL70 & 1 & 24 & B. intestinihominis & 86 & SDCS37 & 7 & 24 & P. ruminicola & 93 \\
\hline SDMOL5 & 1 & 25 & P. shahii & 86 & SDCS44 & 1 & 25 & P. ruminicola & 94 \\
\hline SDMOL21 & 1 & 26 & P. shahii & 88 & SDCS47 & 1 & 26 & P. ruminicola & 94 \\
\hline SDMOL71 & 2 & 27 & P. shahii & 89 & SDCS94 & 1 & 27 & P. ruminicola & 94 \\
\hline SDMOL18 & 1 & 28 & P. shahii & 90 & SDCS11 & 1 & 28 & P. ruminicola & 95 \\
\hline SDMOL30 & 1 & 29 & P. shahii & 90 & SDCS9 & 5 & 29 & P. ruminicola & 95 \\
\hline SDMOL75 & 10 & 30 & P. shahii & 90 & SDCS87 & 2 & 30 & Par. clara & 88 \\
\hline SDMOL76 & 1 & 31 & P. shahii & 90 & SDCS7 & 1 & 31 & Par. clara & 89 \\
\hline SDMOL82 & 2 & 32 & P. shahii & 90 & SDCS60 & 1 & 32 & P. shahii & 85 \\
\hline SDMOL88 & 1 & 33 & P. shahii & 90 & SDCS76 & 2 & 33 & P. shahii & 85 \\
\hline SDMOL109 & 1 & 34 & P. shahii & 90 & SDCS13 & 1 & 34 & P. shahii & 90 \\
\hline SDMOL118 & 1 & 35 & P. shahii & 90 & SDCS86 & 1 & 35 & P. veroralis & 91 \\
\hline SDMOL7 & 1 & 36 & P. bryantii & 90 & SDCS77 & 1 & 36 & P. veroralis & 92 \\
\hline SDMOL28 & 1 & 37 & P. copri & 87 & SDCS104 & 1 & 37 & P. dentalis & 91 \\
\hline SDMOL26 & 1 & 38 & P. copri & 89 & SDCS88 & 1 & 38 & P. albensis & 87 \\
\hline SDMOL135 & 1 & 39 & P. copri & 91 & SDCS21 & 1 & 39 & Ros. hominis & 90 \\
\hline SDMOL34 & 1 & 40 & P. salivae & 89 & SDCS28 & 1 & 40 & Pab. merdae & 84 \\
\hline SDMOL47 & 2 & 41 & P. salivae & 90 & SDCS20 & 8 & 41 & S. dextrinosolvens & 97 \\
\hline SDMOL64 & 3 & 42 & P. salivae & 91 & SDCS89 & 1 & 42 & Rum. bromii & 90 \\
\hline
\end{tabular}


Table 2 Comparison of 16S rRNA gene libraries between the OL and CS groups (Continued)

\begin{tabular}{|c|c|c|c|c|c|c|c|c|c|}
\hline SDMOL74 & 3 & 43 & P. salivae & 91 & SDCS36 & 1 & 43 & Rum. bromii & 95 \\
\hline SDMOL98 & 5 & 44 & P. salivae & 91 & SDCS97 & 1 & 44 & Rum. bromii & 95 \\
\hline SDMOL139 & 3 & 45 & P. salivae & 92 & SDCS38 & 1 & 45 & Pab. merdae & 84 \\
\hline SDMOL63 & 1 & 46 & P. veroralis & 91 & SDCS50 & 1 & 46 & Pro. acetatigenes & 83 \\
\hline SDMOL44 & 16 & 47 & P. veroralis & 92 & SDCS83 & 1 & 47 & A. shahii & 85 \\
\hline SDMOL136 & 16 & 48 & P. veroralis & 92 & SDCS96 & 1 & 48 & Sp. acetigenes & 84 \\
\hline SDMOL53 & 1 & 49 & P. albensis & 91 & SDCS102 & 1 & 49 & C. aldrichii & 86 \\
\hline SDMOL58 & 2 & 50 & P. stercorea & 87 & SDCS105 & 1 & 50 & C. bolteae & 91 \\
\hline SDMOL100 & 1 & 51 & P. multiformis & 91 & & & & & \\
\hline SDMOL117 & 1 & 52 & P. ruminicola & 92 & & & & & \\
\hline SDMOL143 & 1 & 53 & P. dentalis & 91 & & & & & \\
\hline SDMOL31 & 1 & 54 & A. shahii & 85 & & & & & \\
\hline SDMOL37 & 1 & 55 & A. shahii & 88 & & & & & \\
\hline SDMOL127 & 1 & 56 & S. dextrinosolvens & 97 & & & & & \\
\hline SDMOL66 & 1 & 57 & P. brevis & 87 & & & & & \\
\hline
\end{tabular}

P Prevotella, S Succinivibrio, A Alistipes, Par Paraprevotella, Ros Roseburia, Rum Ruminococcus, Sp Sporanaerobacter, C Clostridium, Pab Parabacteroides, Pro Proteiniphilum, B Barnesiella, a number of clones, $b$ sequence indentity, OTU \# OTU No.

groups had 92-96\% sequence similarities with several species belonging to genus Prevotella including P. loescheii, $P$. pleuritidis, P. corporis, P. buccalis, P. dentalis, P. melaninogenica, $P$. salivae, $P$. copri, $P$. denticola, $P$. oulorum and $P$. histicola.

\section{Discussion}

In the present study, two $16 \mathrm{~S}$ rRNA gene libraries and PCR-DGGE were used to study the rumen bacteria in the rumen of domesticated Sika deer feeding on oak leaves-based (OL) and corn stalks-based (CS) diets. Sequences from the two clone libraries and PCR-DGGE bands indicated that the majority of sequences belonged to phylum Bacteroidetes. The findings from the current study are similar to previous findings for other ruminants, such as Reindeer, yaks, cattle and goats [14-18]. The predominance of sequences belonging to the phylum Bacteroidetes highlights their important role in the rumen fermentation of domesticated Sika deer. While, the phylum Firmicutes being prevalent in other ruminants were not found in the OL library [19], which could be caused by the tannins contained in oak leaves, because some studies reported that the tannins in oak leaves may have a negative effect on some of the bacterial species $[20,21]$, and the growth of proteolytic bacteria, such as Butyrivibrio fibrisolvens, Ruminococcus albus and Streptococcus bovis, were inhibited by tannins $[22,23]$. This may also indicate that some species belonging to phylum Firmicutes in the rumen of domestic Sika deer may be sensitive to tannins.

Within the phylum Bacteroidetes, Prevotella-like clones accounted for $97.2 \%$ of the clones in the OL group and
77\% in the CS group. Moreover, the PCR-DGGE results also showed the genus Prevotella represented the predominant bacteria in rumen of domesticated Sika deer (Table 3), which is in agreement with other studies [19,24-28] . The prevalence of Prevotella spp. in rumen fermentation of domesticated Sika deer was likely because they utilize a wide variety of polysaccharides, and are thought to be important contributors to xylan degradation in the rumen [29-32]. Although other studies found that concentrate diets increased the numbers of clones related to Prevotella spp. [33,34], however, in comparison with other ruminants, there was an apparent difference in the proportion of Prevotella spp. [6,25,27,28]. Prevotella spp. belonged to the hydrogen-consuming bacteria, which could produce propionate via succinate or acrylate pathways though fermentation of sugars and lactate, respectively [35-37]. Therefore, the dominant genus Prevotella in the rumen of domesticated Sika deer suggested that the propionate pathway may be relatively vital in the rumen fermentation of domestic Sika deer, which, in turn, may lead to the decreased production of methane, since the succinate-propionate pathway could compete with methanogens for hydrogen [38]. The relationship between Prevotella spp. and methanogens in the rumen of domesticated Sika deer was worth of further investigating. In addition, the bacterial communities in the rumen between domesticated Sika deer, Svalbard reindeer and Norwegian reindeer, all cervids, were compared using Fast UniFrac, which can be used to determine whether communities are significantly different [13]. The results of Principal coordinate analysis (PCoA) between domesticated Sika deer and Reindeer using the Fast 


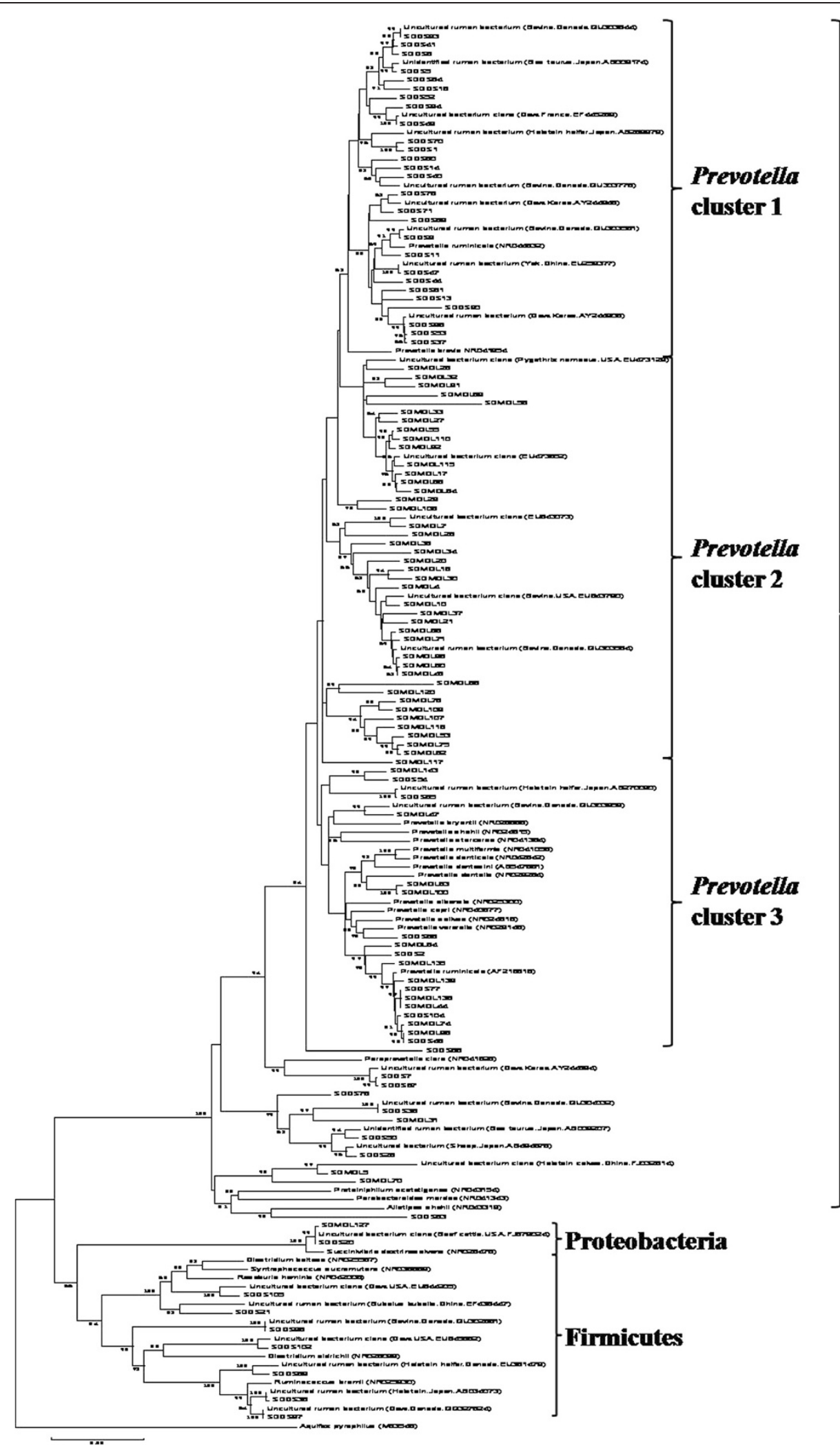

Figure 3 Phylogenetic tree of bacterial 16S rRNA sequences from two groups using the Neighbor-Joining method and Kimura two-parameter model in MEGA. Clones from Sika deer fed oak leaves beginning with SDMOL, followed by clone number, and from corn stalks beginning with SDCS, followed by clone number. Aquifex pyrophilus was used as the outgroup. Statistical significance was verified by bootstrapping 1000 replicates. 


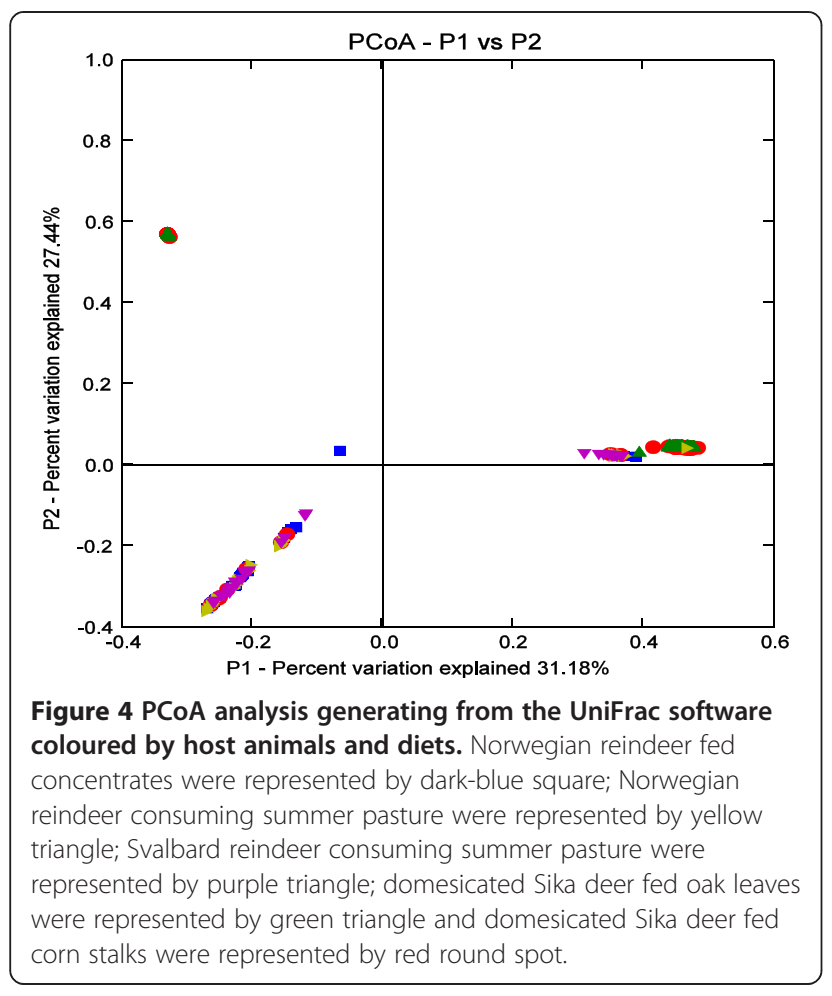

Unifrac platform clearly showed that the rumen bacterial communities were distinct, which can be attributed to the host-species (Figure 5) [13,26,39].

It is important to note, that fibrolytic bacteria, such as $C$. populeti, E. cellulosolvens and Ps. ruminis were discovered in our analysis based on PCR-DGGE, rather than the predominant fibrolytic bacteria, B. fibrisolvens, Fibrobacter succinogenes, Ruminococcus flavefaciens and $R$. albus. This may suggest that the rumen of domesticated Sika deer depend on unique bacterial communities in rumen fermentation. In contrast, the absence of $R$. flavefaciens,
B. fibrisolvens, F. succinogenes and R. albus in the present work may be attributed to the small number of clones may have missed some other members of the bacterial community, and the weak or unidentifiable bands in DGGE. Future work will employ next generation sequencing to effectively elucidate the bacterial diversity present in the rumen of domesticated Sika deer and other livestock. Collectively, these data indicated that the rumen of domesticated Sika deer harbored unique bacterial populations for the fermentation of plant biomass and concentrate diet.

Interestingly, in both clone libraries, none of the sequences were $100 \%$ identical. Rather, most clones were in the range of $83-98 \%$ identify to known species in both libraries. These results suggested that the rumen bacteria of domesticated Sika deer were not previously characterized and that these clones related to Prevotella spp. in the rumen represented new species. This agrees with previous findings suggesting that most of the bacterial species in rumen of other cervids (96\% for Hokkaido Sika deer and 100\% for Svalbard reindeer) are unknown $[26,40]$. Despite the diets and geographic location are important factors affecting bacterial diversity in the rumen, however, the presence of these unknown or unidentified species may be the result of co-evolution between microbial communities and the host.

PCR-DGGE analysis showed that the bacterial diversity in domesticated Sika deer fed corn stalks differed from the domesticated Sika deer consuming oak leaves (Figure 5), indicating forage affected the relative abundance and composition of the bacteria. Moreover, the difference in the Prevotella species between the two groups was very apparent (Table 3). For instance, the results of clone library showed that the proportion of P. ruminicola-like clones (27\%) was abundant in the CS group comparing with those in the OL group, and sequences analysis of PCR-DGGE also indicated that

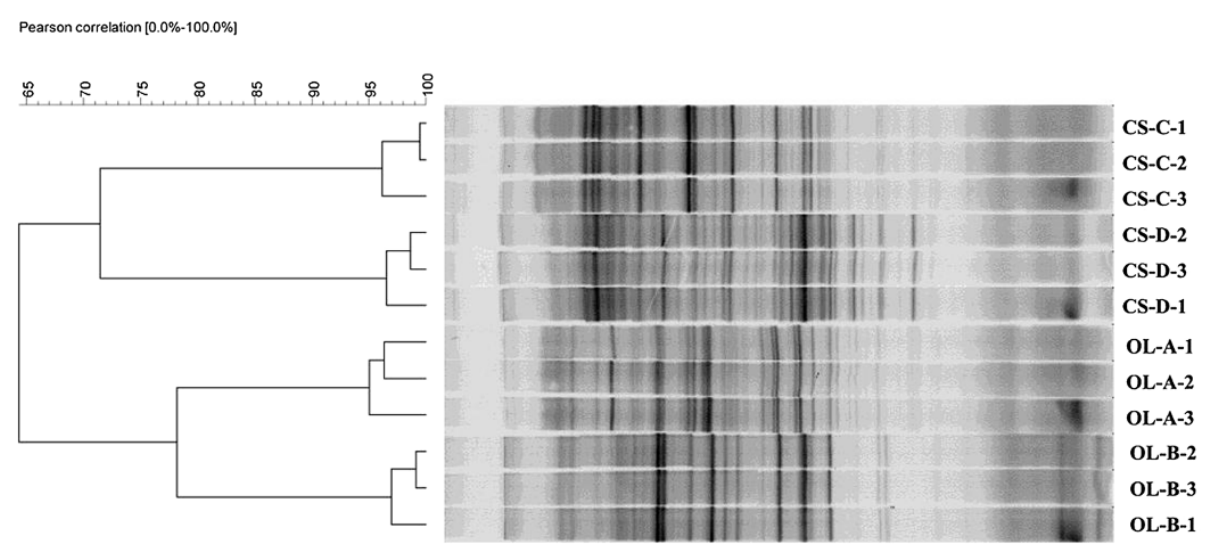

Figure 5 PCR-DGGE profiles of the rumen bacterial 16S rNA gene (V3 region) from domestic Sika deer fed oak leaves (Sika deer A and B) and corn stalks (Sika deer C and D). OL and CS represented Sika deer fed oak leaves and corn stalks, respectively. Three replicates (1,2 and 3) were taken from each Sika deer. Bionumerics software generated the clustering dendrogram using the UPGMA method. 
Table 3 Sequences analysis of V3 region of 16S rRNA gene from PCR-DGGE

\begin{tabular}{|c|c|c|c|c|c|}
\hline \multicolumn{3}{|c|}{ OL group } & \multicolumn{3}{|c|}{ CS group } \\
\hline Band No & Nearest cultured relative (GenBank accession No) & $\%^{\mathrm{a}}$ & Band No & Nearest cultured relative (GenBank accession No) & $\%^{\mathrm{a}}$ \\
\hline O-1 & C. populeti (NR026103) & 99 & $C-1$ & P. ruminicola (NR044632) & 98 \\
\hline $0-2$ & P. salivae (NR024816) & 93 & $C-2$ & P. loescheii (NR043216) & 96 \\
\hline $0-3$ & St. pasteurianus (NR043660) & 100 & $C-3$ & C. populeti (NR026103) & 98 \\
\hline O-4 & P. dentalis (NR029284) & 94 & $C-4$ & P. pleuritidis (NR041541) & 94 \\
\hline $0-5$ & P. salivae (NR024816) & 96 & $C-5$ & C. populeti (NR026103) & 98 \\
\hline $0-6$ & P. denticola (NR042842) & 95 & $C-6$ & P. pleuritidis (NR041541) & 94 \\
\hline $0-7$ & P. oulorum (NR029147) & 94 & C-7 & P. corporis (NR044627) & 94 \\
\hline O-8 & P. buccalis (NR044630) & 94 & C-8 & P. buccalis (NR044630) & 94 \\
\hline O-9 & E. cellulosolvens (NR026106) & 98 & C-9 & P. dentalis (NR029284) & 95 \\
\hline O-10 & S. dextrinosolvens (NR026476) & 98 & $C-10$ & S. dextrinosolvens (NR026476) & 98 \\
\hline O-11 & P. salivae (NR024816) & 95 & $C-11$ & P. dentalis (NR029284) & 93 \\
\hline $0-12$ & M. indoligenes (NR043775) & 97 & $C-12$ & P. melaninogenica (NR042843) & 95 \\
\hline O-13 & Ps. ruminis (NR026315) & 99 & $C-13$ & G. mesophilus (NR041450) & 88 \\
\hline O-14 & P. oulorum (NR029147) & 94 & $C-14$ & E. cellulosolvens (NR026106) & 98 \\
\hline O-15 & P. dentalis (NR029284) & 94 & $C-15$ & P. dentalis (NR029284) & 95 \\
\hline O-16 & P. histicola (NR044407) & 95 & $C-16$ & P. loescheii (NR043216) & 93 \\
\hline O-17 & P. dentalis (NR029284) & 95 & $C-17$ & P. salivae (NR024816) & 88 \\
\hline O-18 & St. pasteurianus (NR043660) & 100 & $\mathrm{C}-18$ & Cp. utactus (NR044049) & 98 \\
\hline O-19 & P. dentalis (NR029284) & 96 & $C-19$ & D. acidaminovorans (NR029034) & 92 \\
\hline \multirow[t]{8}{*}{$0-20$} & P. dentalis (NR029284) & 96 & $C-20$ & D. acidaminovorans (NR029034) & 92 \\
\hline & & & $C-21$ & E. ruminantium (NR024661) & 93 \\
\hline & & & $C-22$ & G. esophilus (NR041450) & 91 \\
\hline & & & $C-23$ & P. copri (NR040877) & 92 \\
\hline & & & $C-24$ & Ca. cynodegmi (NR043063) & 88 \\
\hline & & & $C-25$ & P. copri (NR040877) & 93 \\
\hline & & & $C-26$ & P. dentalis (NR029284) & 94 \\
\hline & & & $C-27$ & B. uniformis (NR040866) & 94 \\
\hline
\end{tabular}

C Clostridium, E Eubacterium, P Prevotella, S Succinivibrio, St Streptococcus, M Moryella, Ps Pseudobutyrivibrio, Cp Coprococcus, G Galbibacter, Ca Capnocytophaga, $B$ Bacteroides, $D$ Dethiosulfovibrio, a sequence similarity.

$P$. ruminicola was only presented in CS group. Interestingly, Prevotella species in the rumen could contribute to cell wall degradation through synergistic interactions with species of cellulolytic bacteria [41]. Therefore, considering the relatively high fiber content (about 36\%) in corn stalks, these $P$. ruminicola-like clones in the CS group may play a role in the degradation of cellulose. This explanation is partly supported by recent metagenomics data from the Svalbard reindeer rumen microbiome, where the presence of polysaccharide utilizing glycoside hydrolase and other carbohydrate-active enzyme families target various polysaccharides including cellulose, xylan and pectin [18].

In the OL group, the distribution of $P$. shahii-like clones (16.5\%), P. veroralis-like clones (23.8\%) and P. salivae-like clones (12.3\%) were several times higher in the OL library than in the CS library, and several bands in the PCR-DGGE analysis showed sequence similarities to P. salivae (Table 3). Previous study reported that $P$. ruminicola may tolerate condensed tannins [22]. Considering the genetic diversity of Prevotella spp. $[27,42]$, it is assumed that the tolerance to tannins of domestic Sika deer may be related to the abundance of Prevotella spp. in the OL group. In addition, we found two bands (O-3 and O-18) were identified as St. pasteurianus using PCR-DGGE. Thus this species may also be important in the process of degrading tannins in diets, because tannin-degrading capability of Streptococcus sp. have been demonstrated in other studies [43-46]. However, these assumptions need to be investigated in future studies.

Phylogenetic analysis indicated the presence of dietspecific subpopulations of Prevotella. Prevotella clusters 1 and 2 not only demonstrated the genetic diversity of Prevotella spp., but also confirmed the above assumption that clones grouped within clusters 1 or 2 may be related to 
the degradation of fiber (cluster 1) or tannins (cluster 2), whereas, the clones in cluster 3 may have common features of degrading starch and proteins contained in concentrate diets (Figure 3). However, clones related to the bacterial genera Sporanaerobacter, Parabacteroides and Proteiniphilum were found in the rumen of domesticated Sika deer fed corn stalks that were not previously reported in the rumen from other ruminants. Sporanaerobacter acetigenes is an acetogenic and a sulfur-reducing bacterium that was isolated from an anaerobic sludge blanket reactor in Mexico $[47,48]$. The rumen has considerable capacity to convert sulfate into sulfur-containing amino acids. Similarly, little is known about Proteiniphilum acetatigenes, which was originally isolated from a UASB reactor treating brewery wastewater in China [49]. These bacteria in rumen of domesticated Sika deer may have other biological functions and is worthy of further investigation.

\section{Conclusions}

In conclusion, this study is the first to report the rumen bacteria in Chinese domesticated Sika deer, consuming either oak leaves-based or corn stalks-based diets. Sequences analysis from 16S rRNA clone libraries and PCR-DGGE revealed that the domesticated Sika deer harbored unique rumen bacterial populations, most of which may present novel species, and that the bacterial compositions were affected by forage. It is speculated that the possible new species of Prevotella may be related to the degradation of tannins or fiber biomass. Moreover, the species diversity of Prevotella sp. in the rumen combined with their synergistic interactions with other microorganisms requires further in depth investigation.

\section{Methods}

\section{Animals and sampling}

Four male rumen-cannulated domestic Sika deer (Cervus nippon) maintained at the research farm $\left(44.04^{\circ} \mathrm{N}\right.$, $129.09^{\circ}$ E) of the Institute of Special Animal and Plant Sciences, Chinese Academy of Agricultural Sciences, in Jilin Province, were used in this study. From September to October, four domestic Sika deer were offered the same concentrated diets $(64.5 \%$ corn, $19.7 \%$ soybean meal, $12.8 \%$ distiller dried grains with solubles and a $3 \%$ mixture of vitamins and mineral salts) and mixed with either oak leaves (OL) or corn stalks (CS). All domestic Sika deer were fed twice each day at 8:00 AM and 4:00 PM and had free access to water. The whole rumen contents, which included solid and fluid fractions, were collected via rumen cannula before the morning feeding, and stored at $-20^{\circ} \mathrm{C}$ for analysis. All domestic Sika deer used in present experiment must be performed according to the animal health and well-being regulations, all animal procedures were approved and authorized by the Chinese Academy of Agricultural Sciences
Animal Care and Use Committee, and by the Wild Animal and Plant Subcommittee, Institute of Special Animal and Plant Sciences.

\section{DNA extraction}

Total DNA was directly extracted from rumen contents containing solid and liquid fraction according to methods described by LaMontagne [50] with few modifications. In brief, $800 \mu$ l lysis buffer $(0.15 \mathrm{M} \mathrm{NaCl}, 0.2 \mathrm{M}$ EDTA, $10 \mathrm{mg} \cdot \mathrm{ml}^{-1}$ lysozyme, pH8.0), $20 \mu \mathrm{l}$ of $20 \mathrm{mg} \cdot \mathrm{ml}^{-1}$ proteinase K (Sigma, Germany), and 0.3 g glass beads (0.1 mm, Sigma, Germany) were added to $0.5 \mathrm{~g}$ of whole rumen contents. After shaking at $37^{\circ} \mathrm{C}$ for $1 \mathrm{~h}, 300 \mu \mathrm{l}$ heated lysis buffer (10\% SDS, $0.1 \mathrm{M} \mathrm{NaCl}, 0.5 \mathrm{M}$ Tris$\mathrm{HCl}, \mathrm{pH} 8.0)$ at $65^{\circ} \mathrm{C}, 300 \mu \mathrm{l}$ phosphate buffer (pH8.0) and $600 \mu \mathrm{l}$ chloroform-isoamyl alcohol $(24: 1, \mathrm{~V} / \mathrm{V})$ were added, and the mixture was incubated at $65^{\circ} \mathrm{C}$ in a water bath for $30 \mathrm{~min}$ with intense shaking $30 \mathrm{~s}$ at $10 \mathrm{~min}$ intervals. After centrifugation at 5,000 rpm for $6 \mathrm{~min}$, the supernatant was transferred to a clean tube. DNA was then precipitated with a 0.6 volume of isopropanol at $-80^{\circ} \mathrm{C}$ for $15 \mathrm{~min}$, and the pellet was washed several times with $75 \%$ ethanol. The DNA was dried and dissolved in TE buffer ( $\mathrm{pH}$ 8.0). The DNA quality was assessed by $0.8 \%$ agarose gel electrophoresis, and the purity was determined by spectrophotometry (SPECORD 50, analytikjena, Germany), after which it was purified using a QIAEX II Gel Extraction Kit (QIAGEN, Germany).

\section{Construction of 16S rRNA gene clone libraries and sequences analyses}

Universal primers 27F (5'-AGAGTTTGATCMTGGC TCAG-3') and 1492R (5'-TACGGYTACCTTGTTACGA CTT-3) were used to amplify the $16 \mathrm{~S}$ rRNA gene (approximately $1.5 \mathrm{~kb}$ ) [51]. Each 50 ul reaction contained 50 ng template DNA, $0.25 \mathrm{mM}$ of each primer, $250 \mathrm{mM}$ dNTPs, $1.25 \mathrm{U}$ of Ex Taq and $5 \mu \mathrm{l} \mathrm{Ex} \mathrm{Taq} \mathrm{buffer}$ (TaKaRa, Dalian). PCR was performed on a 2720 Thermal Cycler (Applied Biosystems, USA) with hot start at $94^{\circ} \mathrm{C}$ for $5 \mathrm{~min}$, followed by 20 cycles of $30 \mathrm{~s}$ at $94^{\circ} \mathrm{C}, 1 \mathrm{~min}$ at $55^{\circ} \mathrm{C}$ and $2 \mathrm{~min}$ at $72^{\circ} \mathrm{C}$; and a final extension at $72^{\circ} \mathrm{C}$ for $10 \mathrm{~min}$. The PCR product was assessed using $2 \%$ agarose gel electrophoresis (approximately $1.5 \mathrm{~kb}$ ), and were purified using a TaKaRa MiniBEST DNA Fragment Purification Kit (TaKaRa, Dalian) and then pooled within each group. Two 16S rRNA gene clone libraries were constructed from the pooled PCR products using the TOPO $^{\circledR}$ TA Cloning ${ }^{\circledR}$ Kit (Invitrogen, USA). Positive (white) clones were screened by colony PCR with the M13 Forward and M13 Reverse primers, and sequenced using an ABI 3730XL DNA Analyzer.

The chimera check program Bellerophon was used to identify chimeric sequences [52]. The remaining sequences were assigned using the Classifier tool available 
at Ribosomal Database Project (RDP) Release 10 at a confidence threshold of $80 \%$ [53]. OTUs based on $97 \%$ sequence identity, and the Shannon-Wiener index-based diversity estimator and the Chaol based index of richness were calculated using MOTHUR platform to determine the diversity and richness of bacterial communities in each group based on the 16S rRNA gene libraries [54]. Libshuff analysis was performed to estimate the similarity between libraries from two diets based on evolutionary distance of all sequences. Coverage and rarefaction curves were also determined using the MOTHUR platform [54]. The 16S rRNA gene sequences were screened using GenBank's BLAST program [55]. The closest related sequences were retrieved and aligned with sequences from the present study using the CLUSTALW 1.83 program in MEGA 5.05 software [56]. A phylogenetic tree was constructed using the Kimura two-parameter model and the Neighbor-Joining method as part of the MEGA 5.05 software. The statistical significance was verified by 1000 bootstrapped replicates. The sequences obtained from this study were submitted to GenBank under the accession numbers JX889268 to JX889378. Furthermore, an unweighted UniFrac distance matrix was constructed from the phylogenetic tree of clone libraries of Norwegian reindeer, Svalbard reindeer and Sika deer, and was visualized using PCoA [13,26,39].

\section{PCR-DGGE banding profiles and statistical analysis}

The variable region (V3) of the bacterial $16 \mathrm{~S}$ rRNA gene was amplified using the primers of F341GC and R534, and PCR condition was described previously [57]. A 40 bp GC-clamp (5'-CGCCCGGGGCGCGCCCCGGGC GGGGCGGGGGCACGGGGGG-3') was on the $5^{\prime}$ end of the F341 primer. The PCR products were loaded onto $8 \%$ polyacrylamide gels $(37.5: 1)$ with a denaturing gradient of $40-60 \%$ at $80 \mathrm{~V}$ over $16 \mathrm{~h}$ at $60^{\circ} \mathrm{C}$. Electrophoresis was performed using Bio-Rad's DCode detection system. The gels were stained with SYBR Green I (Invitrogen, USA) for $25 \mathrm{~min}$ and gel images were captured using the Gel Doc ${ }^{\mathrm{Tm}} \mathrm{XR}^{+}$system (BIO-RAD, CA). Cluster analysis was performed using a Dice similarity coefficient at $0.5 \%$ optimization and $1 \%$ tolerance following the unweighted pair-group method using arithmetic averages (UPGMA) on BioNumerics 6.0 software (AppliedMaths, Kortrijk, Belgium).

Dominant bands were excised from DGGE gel and eluted overnight in $500 \mu \mathrm{l}$ of sterilized $\mathrm{ddH}_{2} \mathrm{O}$ at $4^{\circ} \mathrm{C}$. Extracted DNA was re-amplified using PCR primers F341 and R534 without GC-clamp. The size of PCR products were determined using agarose gel and were purified using QIAquick ${ }^{\circledR}$ PCR Purification Kit (Qiagen, USA). The PCR products were cloned into $\mathrm{TOPO}^{\circledR} \mathrm{TA}$ Cloning ${ }^{\circledR}$ Kit with TOP 10 according to the manufacturer's instruction (Invitrogen, San Diego, CA, USA).
Recombinant plasmids of positive clones (white) were sequenced using ABI 3730XL DNA Analyzer. The sequences were compared with those sequences deposited in NCBI web site using BLAST program [55].

\section{Additional file}

\begin{abstract}
Additional file 1: Dominant bands of PCR-DGGE banding patterns of bacteria 16SrRNA gene (V3 region). In the text, bands from OL group were defined as $\mathrm{O}$ and followed by bands number, bands from CS group begin with $C$ and followed by bands numbers.
\end{abstract}

\section{Abbreviations}

DGGE: Denaturing gradient gel electrophoresis; OTUs: Operational taxonomic units; VFA: Volatile fatty acids; BLAST: Basic local alignment search tool; RDP: Ribosomal database project; PCoA: Principal coordinates analysis.

\section{Competing interests}

The authors declare that they have no competing interests.

\section{Authors' contributions}

ZPL sampled rumen contents, extracted DNA, constructed the clone library, data analysis and drafted the manuscript. ADGW was involved with interpretation of data and with preparing the manuscript. HLL designed the study and drafted the paper. KB, YFY, CX and KYW contributed to sample rumen contents and all of lab works. GYL and FHYconceived the study. All authors read and approved the final manuscript.

\section{Acknowledgements}

Special thanks to Dr. Yanfeng Cheng in the analysis of 165 rRNA gene sequences and Dr. Zhi Gang Cui in the analysis of PCR-DGGE. This work is supported by a Young Researcher funded project (201101086), Science and Technology Development project (20090238) and a Leading Talent and Creative Team project (20121810), all from Jilin province, the Ministry of Agriculture Public Sector (Agriculture) Special Research Project (200903014) and Key Projects in the National Science \& Technology Pillar Program (2011BAI03B02).

\section{Author details}

${ }^{1}$ Department of Economical Animal Nutrition and Feed Science, Institute of Special Animal and Plant Sciences, Chinese Academy of Agricultural Sciences, JiLin, China. ${ }^{2}$ Department of Animal Science, University of Vermont, 570 Main Street, Burlington, VT 05405-0148, USA.

Received: 4 March 2013 Accepted: 1 July 2013

Published: 8 July 2013

\section{References}

1. Hiura T, Hashidoko Y, Kobayashi Y, Tahara S: Effective degradation of tannic acid by immobilized rumen microbes of a sika deer (Cervus nippon yesoensis) in winter. Anim Feed Sci Technol 2010, 155(1):1-8.

2. Clauss M, Lason K, Gehrke J, Lechner-Doll M, Fickel J, Grune T, Jurgen Streich W: Captive roe deer (Capreolus capreolus) select for low amounts of tannic acid but not quebracho: fluctuation of preferences and potential benefits. Comp Biochem Physiol B Biochem Mol Biol 2003, 136(2):369-382.

3. Wright A-DG, Klieve AK: Does the complexity of the rumen microbial ecology preclude methane mitigation? Animal Feed Sci. Technol. 2011, 166-167:248-253.

4. Tajima K, Arai S, Ogata K, Nagamine T, Matsui H, Nakamura M, Aminov Rl, Benno Y: Rumen bacterial community transition during adaptation to high-grain diet. Anaerobe 2000, 6(5):273-284.

5. An DD, Dong XZ, Dong ZY: Prokaryote diversity in the rumen of yak (Bos grunniens) and Jinnan cattle (Bos taurus) estimated by 16S rDNA homology analyses. Anaerobe 2005, 11(4):207-215.

6. Pei CX, Liu QA, Dong CS, Li HQ, Jiang JB, Gao WJ: Diversity and abundance of the bacterial 16S rRNA gene sequences in forestomach of alpacas (Lama pacos) and sheep (Ovis aries). Anaerobe 2010, 16(4):426-432. 
7. Yang LY, Chen J, Cheng XL, Xi DM, Yang SL, Deng WD, Mao HM: Phylogenetic analysis of $16 \mathrm{~S}$ rRNA gene sequences reveals rumen bacterial diversity in Yaks (Bos grunniens). Mol Biol Rep 2010, 37(1):553-562.

8. Aagnes TH, Sormo W, Mathiesen SD: Ruminal microbial digestion in free-living, in captive lichen-ded, and in Starved Reindeer (Rangifer tarandus tarandus) in winter. Appl Environ Microbiol 1995, 61(2):583-591.

9. Edwards JE, McEwan NR, Travis AJ, Wallace RJ: 16S rDNA library-based analysis of ruminal bacterial diversity. Antonie Leeuwenhoek Int J Gen Mo Microbiol 2004, 86(3):263-281.

10. Ichimura Y, Yamano H, Takano T, Koike S, Kobayashi Y, Tanaka K, Ozaki N, Suzuki M, Okada H, Yamanaka M: Rumen microbes and fermentation of wild sika deer on the Shiretoko peninsula of Hokkaido Island, Japan. Ecol Res 2004, 19(4):389-395.

11. Kocherginskaya SA, Aminov RI, White BA: Analysis of the rumen bacterial diversity under two different diet conditions using denaturing gradient gel electrophoresis, random sequencing, and statistical ecology approaches. Anaerobe 2001, 7(3):119-134.

12. Shi PJ, Meng K, Zhou ZG, Wang YR, Diao QY, Yao B: The host species affects the microbial community in the goat rumen. Lett Appl Microbiol 2008, 46(1):132-135.

13. Lozupone C, Knight R: UniFrac: a new phylogenetic method for comparing microbial communities. Appl Environ Microbiol 2005, 71(12):8228-8235.

14. Cho SJ, Cho KM, Shin EC, Lim WJ, Hong SY, Choi BR, Kang JM, Lee SM, Kim $\mathrm{YH}$, Kim H, et al: 16S rDNA analysis of bacterial diversity in three fractions of cow rumen. J Microbiol Biotechnol 2006, 16(1):92-101.

15. Yang SL, Ma SC, Chen J, Mao HM, He YD, Xi DM, Yang LY, He TB, Deng WD: Bacterial diversity in the rumen of Gayals (Bos frontalis), Swamp buffaloes (Bubalus bubalis) and Holstein cow as revealed by cloned $16 \mathrm{~S}$ rRNA gene sequences. Mol Biol Rep 2010, 37(4):2063-2073.

16. Cunha IS, Barreto CC, Costa OYA, Bomfim MA, Castro AP, Kruger RH, Quirino BF: Bacteria and archaea community structure in the rumen microbiome of goats (Capra hircus) from the semiarid region of Brazil. Anaerobe 2011, 17(3):118-124

17. Li MJ, Zhou M, Adamowicz E, Basarab JA, Guan LL: Characterization of bovine ruminal epithelial bacterial communities using 16S rRNA sequencing, PCR-DGGE, and qRT-PCR analysis. Vet Microbio/ 2012, 155(1):72-80.

18. Pope PB, Mackenzie AK, Gregor I, Smith W, Sundset MA, McHardy AC, Morrison M, Eijsink VG: Metagenomics of the Svalbard reindeer rumen microbiome reveals abundance of polysaccharide utilization loci. PLoS One 2012, 7(6):e38571.

19. Kim M, Morrison M, Yu Z: Status of the phylogenetic diversity census of ruminal microbiomes. FEMS Microbiol Ecol 2011, 76(1):49-63.

20. Bae HD, McAllister TA, Yanke J, Cheng KJ, Muir AD: Effects of condensed tannins on endoglucanase activity and filter paper digestion by Fibrobacter succinogenes S85. Appl Environ Microbiol 1993, 59(7):2132-2138.

21. McSweeney CS, Palmer B, McNeill DM, Krause DO: Microbial interactions with tannins: nutritional consequences for ruminants. Anim Feed Sci Technol 2001, 91(1-2):83-93.

22. Jones GA, MCAllister TA, Muir AD, Cheng KJ: Effects of sainfoin (Onobrychis viciifolia Scop.) condensed tannins on growth and proteolysis by four strains of ruminal bacteria. App/ Environ Microbiol 1994, 60(4):1374-1378

23. Min BR, Attwood GT, McNabb WC, Molan AL, Barry TN: The effect of condensed tannins from Lotus corniculatus on the proteolytic activities and growth of rumen bacteria. Anim Feed Sci Technol 2005, 121(1-2):45-58

24. Koike S, Yoshitani S, Kobayashi Y, Tanaka K: Phylogenetic analysis of fiber-associated rumen bacterial community and PCR detection of uncultured bacteria. FEMS Microbiol Lett 2003, 229(1):23-30.

25. Stevenson DM, Weimer PJ: Dominance of Prevotella and low abundance of classical ruminal bacterial species in the bovine rumen revealed by relative quantification real-time PCR. App/ Microbiol Biotechnol 2007, 75(1):165-174.

26. Sundset MA, Praesteng KE, Cann IK, Mathiesen SD, Mackie RI: Novel rumen bacterial diversity in two geographically separated sub-species of reindeer. Microb Ecol 2007, 54(3):424-438.

27. Bekele AZ, Koike S, Kobayashi Y: Genetic diversity and diet specificity of ruminal Prevotella revealed by $16 \mathrm{~S}$ rRNA gene-based analysis. FEMS Microbiol Lett 2010, 305(1):49-57.
28. Wu S, Baldwin RL, Li W, Li C, Connor EE, Li RW: The bacterial community composition of the bovine rumen detected using pyrosequencing of 16S rRNA genes. Metagenomics 2012, 1:1-11.

29. Cotta MA: Interaction of ruminal bacteria in the production and utilization of maltooligosaccharides from starch. App/ Environ Microbiol 1992, 58(1):48-54.

30. Gardner RG, Wells JE, Russell JB, Wilson DB: The cellular location of Prevotella ruminicola beta-1,4-D-endoglucanase and its occurrence in other strains of ruminal bacteria. Appl Environ Microbiol 1995, 61(9):3288-3292.

31. Matsui H, Ogata K, Tajima K, Nakamura M, Nagamine T, Aminov Rl, Benno Y: Phenotypic characterization of polysaccharidases produced by four Prevotella type strains. Curr Microbiol 2000, 41(1):45-49.

32. Krause DO, Denman SE, Mackie RI, Morrison M, Rae AL, Attwood GT, McSweeney CS: Opportunities to improve fiber degradation in the rumen: microbiology, ecology, and genomics. FEMS Microbiol Rev 2003, 27(5):663-693.

33. Fernando SC, Purvis HT, Najar FZ, Sukharnikov LO, Krehbiel CR, Nagaraja TG, Roe BA, DeSilva U: Rumen microbial population dynamics during adaptation to a high-grain diet. Appl Environ Microbiol 2010, 76(22):7482-7490.

34. Sadet-Bourgeteau S, Martin C, Morgavi DP: Bacterial diversity dynamics in rumen epithelium of wethers fed forage and mixed concentrate forage diets. Vet Microbio/ 2010, 146(1-2):98-104.

35. Strobel HJ: Vitamin B12-dependent propionate production by the ruminal bacterium Prevotella ruminicola 23. Appl Environ Microbiol 1992, 58(7):2331-2333.

36. Purushe J, Fouts DE, Morrison M, White BA, Mackie Rl, North American Consortium for Rumen B, Coutinho PM, Henrissat B, Nelson KE: Comparative genome analysis of Prevotella ruminicola and Prevotella bryantii: insights into their environmental niche. Microb Ecol 2010, 60(4):721-729.

37. Flint HJ, Bayer EA, Rincon MT, Lamed R, White BA: Polysaccharide utilization by gut bacteria: potential for new insights from genomic analysis. Nat Rev Microbiol 2008, 6(2):121-131.

38. Newbold CJ, Lopez S, Nelson N, Ouda JO, Wallace RJ, Moss AR: Propionate precursors and other metabolic intermediates as possible alternative electron acceptors to methanogenesis in ruminal fermentation in vitro. Br J Nutr 2005, 94(1):27-35.

39. Sundset M, Edwards J, Cheng Y, Senosiain R, Fraile M, Northwood K, Praesteng KE, Glad T, Mathiesen S, Wright AD: Molecular diversity of the rumen microbiome of Norwegian Reindeer on natural summer pasture. Microb Ecol 2009, 57(2):335-348.

40. Kobayashi $Y$ : Inclusion of novel bacteria in rumen microbiology: need for basic and applied science. Anim Sci J 2006, 77(4):375-385.

41. Whitehead TR: Analyses of the gene and amino acid sequence of the Prevotella (Bacteroides) ruminicola 23 xylanase reveals unexpected homology with endoglucanases from other genera of bacteria. Curr Microbiol 1993, 27(1):27-33.

42. Ramsak A, Peterka M, Tajima K, Martin JC, Wood J, Johnston MEA, Aminov Rl, Flint HJ, Avgustin G: Unravelling the genetic diversity of ruminal bacteria belonging to the CFB phylum. FEMS Microbiol Ecol 2000, 33(1):69-79.

43. Brooker JD, O'Donovan LA, Skene I, Clarke K, Blackall L, Muslera P: Streptococcus caprinus sp. nov, a tannin-resistant ruminal bacterium from feral goats. Lett Appl Microbiol 1994, 18(6):313-318.

44. Sly LI, Cahill MM, Osawa R, Fujisawa T: The tannin-degrading species Streptococcus gallolyticus and Streptococcus caprinus are subjective synonyms. Int J Syst Bacteriol 1997, 47(3):893-894.

45. Chamkha M, Patel BK, Traore A, Garcia JL, Labat M: Isolation from a shea cake digester of a tannin-degrading Streptococcus gallolyticus strain that decarboxylates protocatechuic and hydroxycinnamic acids, and emendation of the species. Int J Syst Evol Microbiol 2002, 52(Pt 3):939-944.

46. Goel G, Puniya AK, Singh K: Tannic acid resistance in ruminal streptococcal isolates. J Basic Microbiol 2005, 45(3):243-245.

47. Eaton HL, De Lorme M, Chaney RL, Craig AM: Ovine ruminal microbes are capable of biotransforming hexahydro-1,3,5-trinitro-1,3,5-triazine (RDX). Microb Ecol 2011, 62(2):274-286.

48. Hernandez-Eugenio G, Fardeau ML, Cayol JL, Patel BK, Thomas P, Macarie H, Garcia JL, Ollivierx P: Sporanaerobacter acetigenes gen. nov., sp. nov., a 
novel acetogenic, facultatively sulfur-reducing bacterium. Int J Syst Evol Microbiol 2002, 52(Pt 4):1217-1223.

49. Chen S, Dong X: Proteiniphilum acetatigenes gen. nov., sp. nov., from a UASB reactor treating brewery wastewater. Int I Syst Evol Microbiol 2005, 55(Pt 6):2257-2261.

50. LaMontagne MG, Michel FC, Holden PA, Reddy CA: Evaluation of extraction and purification methods for obtaining PCR-amplifiable DNA from compost for microbial community analysis. J Microbiol Methods 2002, 49(3):255-264

51. Lane DJ: $16 \mathrm{~S} / 23 \mathrm{~S}$ rRNA sequencing. In Nucleic acid techniques in bacteria systematics. Edited by Stackebrandt EGM. New York: Wiley; 1991:115-175.

52. Huber T, Faulkner G, Hugenholtz P: Bellerophon: a program to detect chimeric sequences in multiple sequence alignments. Bioinformatics 2004, 20(14):2317-2319.

53. Cole JR, Wang Q, Cardenas E, Fish J, Chai B, Farris RJ, Kulam-Syed-Mohideen AS, McGarrell DM, Marsh T, Garrity GM, et al: The Ribosomal Database Project: improved alignments and new tools for rRNA analysis. Nucleic Acids Res 2009, 37:D141-D145.

54. Schloss PD, Westcott SL, Ryabin T, Hall JR, Hartmann M, Hollister EB, Lesniewski RA, Oakley BB, Parks DH, Robinson CJ, et al: Introducing mothur: open-source, platform-independent, community-supported software for describing and comparing microbial communities. Appl Environ Microbiol 2009, 75(23):7537-7541.

55. Altschul SF, Madden TL, Schaffer AA, Zhang JH, Zhang Z, Miller W, Lipman DJ: Gapped BLAST and PSI-BLAST: a new generation of protein database search programs. Nucleic Acids Res 1997, 25(17):3389-3402.

56. Tamura K, Peterson D, Peterson N, Stecher G, Nei M, Kumar S: MEGA5: molecular evolutionary genetics analysis using maximum likelihood, evolutionary distance, and maximum parsimony methods. Mol Biol Evol 2011, 28(10):2731-2739.

57. Muyzer G, de Waal EC, Uitterlinden AG: Profiling of complex microbial populations by denaturing gradient gel electrophoresis analysis of polymerase chain reaction-amplified genes coding for $16 \mathrm{~S}$ rRNA. Appl Environ Microbiol 1993, 59(3):695-700.

doi:10.1186/1471-2180-13-151

Cite this article as: Li et al:: Molecular diversity of rumen bacterial communities from tannin-rich and fiber-rich forage fed domestic Sika deer (Cervus nippon) in China. BMC Microbiology 2013 13:151.

\section{Submit your next manuscript to BioMed Central and take full advantage of:}

- Convenient online submission

- Thorough peer review

- No space constraints or color figure charges

- Immediate publication on acceptance

- Inclusion in PubMed, CAS, Scopus and Google Scholar

- Research which is freely available for redistribution 